\title{
Photothermal Circular Dichroism of Single Nanoparticles Rejecting Linear Dichroism by Dual Modulation
}

\author{
Patrick Spaeth, ${ }^{\ddagger}$ Subhasis Adhikari, ${ }^{\ddagger}$ Martin Dieter Baaske, Sergii Pud, Jacco Ton, and Michel Orrit*
}

Cite This: ACS Nano 2021, 15, 16277-16285

Read Online

ACCESS | Lلll Metrics \& More | 回 Article Recommendations | st Supporting Information

ABSTRACT: Circular dichroism (CD) is the property of chiral nanoobjects to absorb circularly polarized light of either handedness to different extents. Photothermal microscopy enables the detection of $C D$ signals with high sensitivity and provides a direct absorptive response of the samples under study. To achieve CD measurements at the single-particle level, one must reduce such artifacts as leakage of linear dichroism (LD) and residual intensity modulation. We have simulated our setup with a simple model, which allows us to tune modulation parameters to obtain a CD signal virtually free from artifacts. We demonstrate the sensitivity of our setup by measuring the very weak inherent $C D$ signals of single gold nanospheres. We furthermore demonstrate that our method can be extended to obtain spectra of the full absorptive properties of single nanoparticles, including isotropic absorption, linear dichroism, and circular dichroism. We then

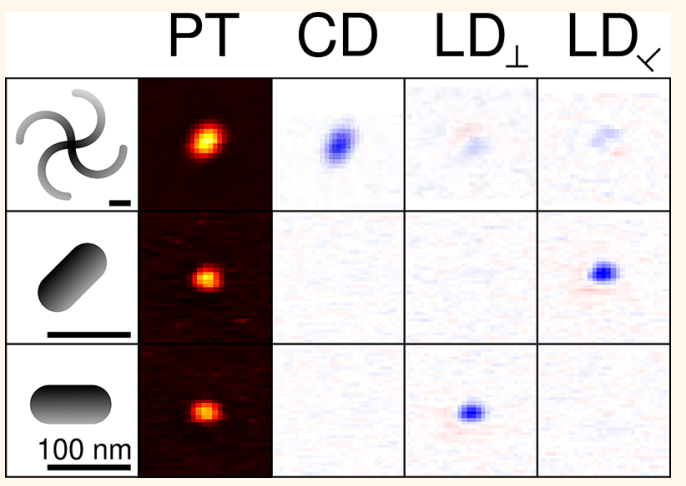
investigate nominally achiral gold nanoparticles immersed in a chiral liquid. Carefully taking into account the intrinsic chirality of the particles and its change due to heat-induced reshaping, we find that the chiral liquid carvone surrounding the particle has no measurable effect on the particles' chirality, down to $g$-factors of $3 \times 10^{-4}$.

KEYWORDS: photothermal microscopy, chirality, circular dichroism, nanoparticles, nanophotonics, single-particle spectroscopy, linear dichroism

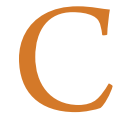
ircular dichroism (CD) is exhibited by chiral molecules or structures that display different absorption for circular polarized light of either handedness. This absorptive property provides valuable structural information about the molecules or the objects under study. The dispersive counterpart of $\mathrm{CD}$ is called circular birefringence (CB) and is considered less often, although the $\mathrm{CB}$ and $\mathrm{CD}$ spectra, being related by a Kramers-Kronig relation, ${ }^{1,2}$ have the same physical content. Several other groups have specifically designed plasmonic and dielectric structures to enhance the weak light-molecule interaction, ${ }^{3-9}$ either by enhancing the local field or by enhancing the helicity of the field. Both chirality enhancement approaches lead to an increased differential absorption ( $g$-factor). The ultimate goal of both approaches, field or helicity enhancement, is to eventually sense chirality ( $\mathrm{CD}$ and $\mathrm{CB}$ ) at the single-molecule level. However, this very ambitious goal is still extremely remote. Here, we propose to improve the sensitivity of pure $\mathrm{CD}$ measurements of single nanoparticles in an optical microscope. CD is typically measured on large ensembles of the analytes of interest, in transmission through a solution. The light polarization is modulated at high frequencies and the polarization-dependent transmission is analyzed with a lock-in amplifier. Commercial CD spectro-polarimeters operate according to a dual-polarization modulation scheme, described by Jellison et al. ${ }^{10,11}$ This scheme facilitates the simultaneous measurement of multiple optical properties. This method is a standard way to obtain CD spectra of drugs and biomolecules including proteins, providing a sensitivity to differential absorptions as low as $10^{-5}$. However, due to their requirement of large volumes of analyte, these spectro-polarimeters naturally lack spatial resolution and therefore are not suitable to perform $\mathrm{CD}$ measurements on single nanoparticles or even on minute quantities of biomolecules. In bulk measurements on solutions, the linear dichroism (LD), i.e., the differential absorption of individual molecules or particles for light

Received: June 23, 2021

Published: September 22, 2021 


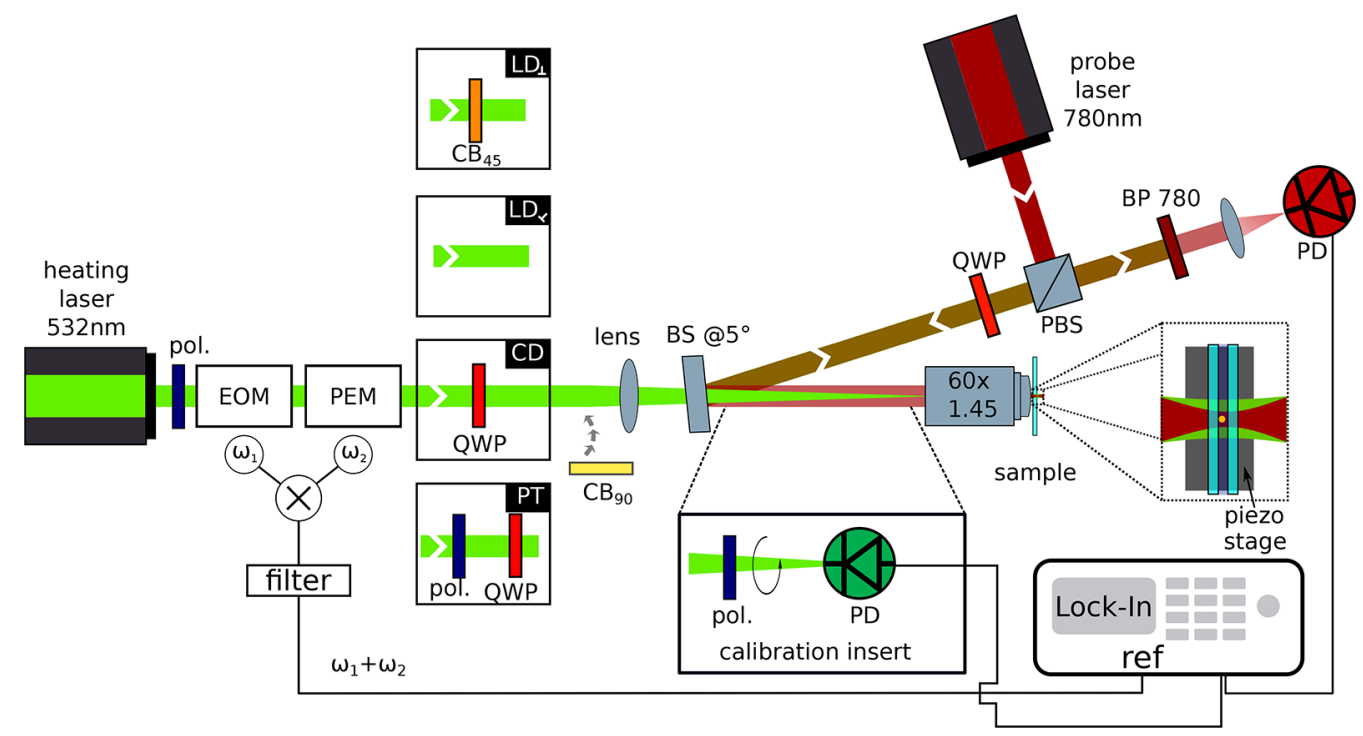

Figure 1. Schematic representation of the dual modulation PTCD setup with the additional calibration insert, where a rotatable calibration polarizer and photodiode (PD) can be inserted into the heating beam path. By flipping a set of different polarization components, i.e., circular birefringence plates and polarizer, we are able to measure all absorptive properties, $C D, L_{\perp}$, and $L D_{\times}$, as well as the isotropic absorption of the sample. The $532 \mathrm{~nm}$ heating laser is passed through a polarizer and two polarization modulators, the EOM and the PEM. To measure PT, CD, and both LDs, we insert different sets of polarization components (polarizer, quarter-wave plate (QWP), and circular birefringent plate $C_{45}$ which rotates the linear polarization by $45^{\circ}$ ). The circular birefringent plate $\mathrm{CB}_{90}$ which rotates any linear polarization by $90^{\circ}$ is used to check the leakage of $L D$ into $C D$. The heating laser is focused at the back focal plane of the objective by means of a wide-field lens (focal length $50 \mathrm{~cm}$ ) in a Koehler scheme, to illuminate a sample area of about $20 \mu \mathrm{m}^{2}$. The $780 \mathrm{~nm} \mathrm{CW}$ probe laser is used in a cat's eye configuration (i.e., a combination of a polarizing beam splitter (PBS) and a QWP). The probe laser is reflected at a 50/50 beam splitter (BS) at an angle of $5^{\circ}$ and focused in the sample plane using a high-NA oil-immersion objective $(60 \times, \mathrm{NA}=1.45)$. The sample is scanned using a piezo stage. The reflected probe beam at the glass-oil interface interferes with the probe beam scattered by the thermal lens. The interference signal is filtered from the heating laser using a band-pass filter (BP 780) and focused onto a photodiode connected with a lock-in amplifier. A frequency mixer is used to add the two frequencies used for modulation of the two modulators and send the sum frequency to the lock-in as a reference.

polarized linearly in different directions naturally vanishes in the orientational average, unlike their $\mathrm{CD}$. At the singleparticle level, however, LD can be very strong because of shape-dependent plasmon resonances. Therefore, conventional $\mathrm{CD}$ spectroscopy is difficult to apply to single LD-active particles or to small ensembles thereof, because of cross talk between $\mathrm{LD}$ and $\mathrm{CD}$ signals, hereafter referred to as LD-to-CD leakage.

An extinction-based approach to $\mathrm{CD}$ measurements of single nanoparticles was proposed by Markovich and co-workers. ${ }^{12}$ In their scheme, the probing beam must fulfill very strict polarization requirements, not only on the excitation path but also on the detection path. A single-laser method that aims at high spatial resolution requires strongly focused light and thereby large-NA objectives. Under these tight-focusing conditions, the polarization distribution in the focus plane is complex, ${ }^{13}$ due to contributions of the large span of angles necessary to produce a diffraction-limited focus. Moreover, the high NA renders the technique prone to artifacts owed to tiny imperfections of the beam alignment. ${ }^{14}$ Any offset of the beam from the center of the objective will lead to a difference in the intensity distribution in the focus for different polarizations. This difference can easily be as large as $10^{-2}$ in relative intensity units, even for small beam deviations. Thus, these artifacts can easily dominate typical CD values.

Recently, we proposed a photothermal microscopic technique to image $\mathrm{CD}^{15}$ which, thanks to its two-color scheme, separates the two conflicting requirements of polarization purity and high spatial resolution. As the heating beam is only weakly focused, due to Koehler illumination, there are no large angles in the focus plane, which greatly facilitates high polarization purity in the focus. At the same time, the strongly focused probe beam that only senses heating effects ensures a high spatial resolution. Additionally, because it relies on the photothermal effect, the technique provides the direct absorptive response of the sample, free from scattering and birefringence contributions. Our first demonstration experiment, ${ }^{15}$ despite its high sensitivity, was prone to a number of artifacts due to the single, square-wave modulation scheme of the polarization of the heating beam. Herein, we propose a series of improvements to remedy these defects.

A first artifact that must be eliminated is residual intensity modulation of the probe or pump beam that possibly occurs at the measurement frequency of CD. Herein, we propose a dualmodulation method using two sinusoidally driven polarization modulators, a photoelastic modulator (PEM) and an electrooptical modulator (EOM). The choice of at least one EOM is convenient to fulfill the requirement of a tunable bias retardation, whose importance is explained in the Supporting Information. The mechanical oscillation of the piezoelectric actuators of the PEM can cause tiny vibrations at the PEM modulation frequency and lead to residual modulation of the collected probe light intensity and to a background signal. Likewise, the EOM exhibits a slight residual intensity modulation on top of the polarization modulation, due to photoelastic scattering. This effect, also referred to as residual amplitude modulation, ${ }^{16,17}$ leads to undesired intensity modulation of the heating beam at the driving frequency of 
a)

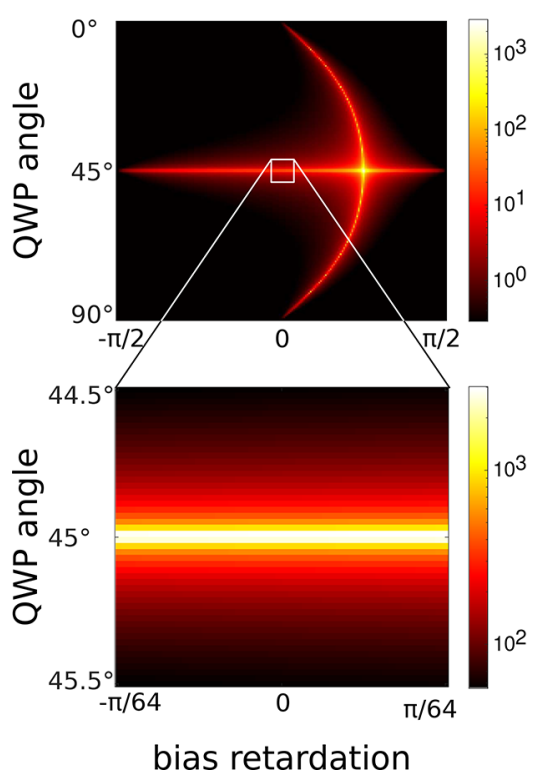

b)

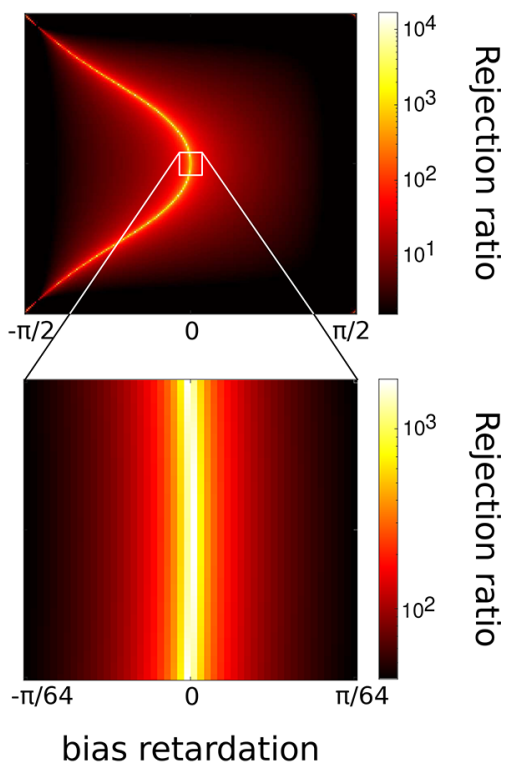

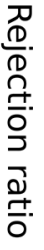

Figure 2. Calculated rejection ratio of $(a) \mathrm{LD}_{\perp}$ and $(\mathrm{b}) \mathrm{LD}_{\times}$as a function of EOM bias retardation and $\mathrm{QWP}$ orientation. The zoom-in in the lower panel shows that in the region where $\mathrm{LD}_{\perp}$ and $\mathrm{LD}_{\times}$are both large the rejection ratio is only dependent on one parameter, either bias voltage for $\mathrm{LD}_{\perp}$ or QWP orientation for $\mathrm{LD}_{\times}$.

the EOM modulator. Both the PEM's and the EOM's intensity modulations will cause artifact $\mathrm{CD}$ signals for any absorbing sample if $\mathrm{CD}$ is measured directly at the driving frequency of the individual modulators.

A second artifact that has to be taken care of is the possible cross talk between LD and CD effects. ${ }^{11,18-21}$ This artifact is mainly induced by static linear birefringence of the optical components and in the special case of quarter-wave plates (QWP) by deviations from the perfect $\pi / 2$ retardation. When performing measurements of ensembles of isotropically distributed molecules or particles in solution, linear dichroic effects can be neglected thanks to ensemble averaging. When performing $\mathrm{CD}$ studies of individual plasmonic particles, it is crucial to avoid this cross talk as the LD of plasmonic particles can easily exceed their CD by some orders of magnitude. Any sample exhibiting LD can cause an artifact signal in a CD measurement. When LD-to-CD leakage is not carefully removed, it can lead to erroneous interpretations of the results. The reverse effect, $\mathrm{CD}$-to-LD leakage, although present in principle, can be neglected as long as $\mathrm{CD}$ is much weaker than LD.

To solve all problems of residual intensity modulation and of the cross-talk between $\mathrm{CD}$ and $\mathrm{LD}$, we apply a purely sinusoidal modulation scheme based on the design of Jellison et al. ${ }^{11}$ We insert an additional QWP on the heating beam path. This QWP shifts the CD signal to the sum $\omega_{2}+\omega_{1}$ and the difference $\omega_{2}-\omega_{1}$ frequencies of the two modulators. We perform lock-in detection at one of these two frequencies. We have chosen the sum frequency, because we expect less $1 / f$ noise at higher frequencies. More importantly, at these combination frequencies the small residual intensity modulations of the two modulators become second-order effects which can be safely neglected.

Furthermore, if we choose the first modulator to be the EOM, then we find that the cross talk of LD into CD induced by imperfections of the optical components, such as residual static birefringence exhibited by EOM, PEM, or QWP, can be compensated in a straightforward manner by combining a slight rotation of the QWP with tuning of the bias voltage of the EOM.

Figure 1 illustrates our imaging photothermal microscope with dual polarization modulation. The setup is not only capable of measuring circular dichroism but furthermore has the capability to measure linear dichroism and the full absorption of one of the circular polarizations. Together with $\mathrm{CD}$, this measurement provides the isotropic absorption that could be measured in an unpolarized photothermal experiment. It is important to stress here that, to fully retrieve the LD information, at least two measurements have to be performed, one in the $\left[0^{\circ} ; 90^{\circ}\right]$ basis and one in the $\left[+45^{\circ} ;-45^{\circ}\right]$ basis. We abbreviate the former with $\mathrm{LD}_{\perp}$ and the latter with $\mathrm{LD}_{\times}$ (noted with a tilted $\perp$ subscript in all figures and in the Supporting Information).

The heating beam $(532 \mathrm{~nm})$ is polarization-modulated by the EOM and the PEM in series. It then passes a variable set of polarization optics that define the operation mode of the setup: (i) $\mathrm{LD}_{\times}$-sensitive without any additional polarization element, (ii) $\mathrm{LD}_{\perp}$-sensitive with a $45^{\circ}$ circular birefringent plate (or $45^{\circ}$ polarization rotator), (iii) CD-sensitive with QWP alone, and (iv) photothermal absorption-sensitive (PT) with a linear polarizer $\left(0^{\circ}\right)$ and QWP. The QWP placed after the polarizer practically provides the isotropic absorption signal in the usual case of particles with very weak CD. Illumination with this intensity-modulated, circularly polarized heating beam provides us with a signal which is very close to the regular photothermal signal and therefore allows us to normalize the $\mathrm{CD}$ and LD signals to the isotropic absorption of the particle. In this manner, we calculate the $g$-factor of a sample by simply dividing the $\mathrm{CD}$ or $\mathrm{LD}$ measurement with the respective PT measurement:

$$
g_{\mathrm{CD}(\mathrm{LD})}=\frac{\mathrm{CD}(\mathrm{LD})}{\mathrm{PT}}
$$


a)

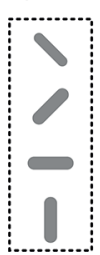

b) $\quad \mathrm{LD}_{<}$

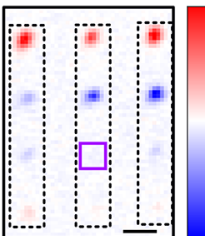

c)

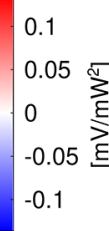

$\mathrm{LD}_{\perp}$

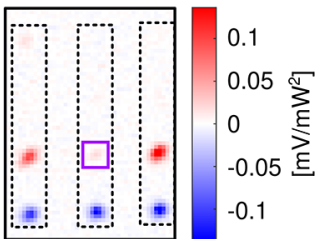

d) $\quad C D$

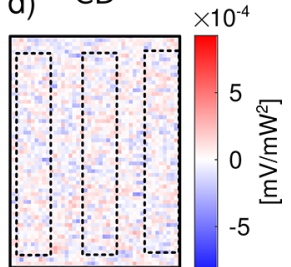

Figure 3. LD and CD scans of 12 aluminum NRs (size $100 \times 40 \mathrm{~nm}^{2}$, thickness $60 \mathrm{~nm}$ ) exhibiting strong linear dichroism. (a) illustrates the spatial orientation of the rods. $(\mathrm{b}-\mathrm{d}) \mathrm{LD}_{\times}, \mathrm{LD}_{\perp}$, and $\mathrm{CD}$ scans, respectively. The phase-sensitive detection enables the determination of the NRs absolute orientation via the amplitudes' sign. The absence of any observable signal in the CD measurement (d) proves the strong rejection of $L D$ signals. The weaker residual signals in the $L D_{\times}$and $L D_{\perp}$ scans are due to a slight misalignment of the sample with respect to the polarization modulators' axes. The square box indicates a nanorod with a reduced absorption due to a fabrication fault. The scale bar is 2 $\mu \mathrm{m}$. The probe beam intensity was $2 \mathrm{~mW}$ and the heating beam intensity was $11 \mathrm{~mW}$, measured at the entrance of the objective.

The respective FFT spectra of signals in the different measurement modes are shown in Figures S2-S4. For objects with strong LD signals, a $90^{\circ}$ circular birefringent plate can be added, $^{22}$ to further improve the cancellation of LD-to-CD leakage and other LD-related artifacts. The heating beam is weakly focused on the sample $(5 \mu \mathrm{m}$ in the focal plane, corresponding to an effective NA of 0.07 ), whereas the probe beam (at $780 \mathrm{~nm}$ ) is tightly focused to retain a high spatial resolution. The reflected probe beam is then cleaned up by removing any residual heating light by means of a long-pass filter and sent to a photodiode for detection. A more detailed description of the setup and the purpose of the $90^{\circ}$-circular birefringent plate can be found in the Supporting Information.

\section{RESULTS AND DISCUSSION}

Simulations. To optimize its performance, we modeled our setup by means of Jones matrix calculus and performed simulations to choose the optimal modulation parameters and to estimate the setup's sensitivity to misalignment and imperfections of its optical components. We want to point out here that there is no need to perform a full Mueller matrix analysis as the weakly focused heating beam $(\sim 5 \mu \mathrm{m})$ is free from the various depolarizing effects that can occur in tightly focused beams and in transmission methods. ${ }^{20}$

In an ideal setup, i.e., if we were only sensitive to $C D$, then we expect zero modulation depth at the sum (or difference) frequency when looking at purely linear dichroic samples. Due to imperfections of the optical elements, however, we have to anticipate cross talk between $C D$ and LD. To quantify this cross talk, we calculate the rejection ratio $R_{\text {ext }}=\left|\mathrm{LD}\left(\omega_{1}+\omega_{2}\right)\right| / \operatorname{ICD}\left(\omega_{1}+\omega_{2}\right) \mid$ that we define as the ratio of the absolute value of the $\mathrm{LD}$, divided by the absolute value of the spurious CD signal measured in the CD channel for a nanoparticle with pure LD absorption, without any intrinsic $\mathrm{CD}$. We calculate that ratio at the sum frequency of the two modulators and under the assumption that the absorption asymmetry ( $g$-factor) is the same for three ideal samples showing pure $\mathrm{LD}_{\perp}, \mathrm{LD}_{\times}$, and $\mathrm{CD}$. Unlike other dual modulation approaches, ${ }^{11,23}$ we keep the amplitudes of the phase modulation of the two modulators fixed (both $\pi / 2$ ) and explore the effect of changing the EOM bias retardation and the QWP's fast axis orientation on the rejection ratio of $\mathrm{LD}_{\perp}$ and $\mathrm{LD}_{\times}$. Our goal is to find a set of modulation parameters that optimizes this rejection ratio and thereby reduces the cross talk between $\mathrm{LD}_{\perp}$ or $\mathrm{LD}_{\times}$and $\mathrm{CD}$. In our model, we take into account imperfections of all retarding elements, including the QWP and the bias and amplitude of retardation by the PEM and the EOM. The detailed mathematical representation is discussed in the Supporting Information.

Figure 2 shows a map of the calculated rejection ratio in our dual-modulation configuration as a function of EOM bias retardation (static linear birefringence) and QWP's orientation. Large rejection ratios for both $\mathrm{LD}_{\perp}$ and $\mathrm{LD}_{\times}$are important, especially when measuring anisotropic plasmonic particles, e.g., quasi-spherical nanoparticles (NPs) and nanorods (NRs), that can exhibit linear dichroism which is up to 2 orders of magnitude stronger than their circular dichroism. The lower left panel of Figure 2 shows that the $\mathrm{LD}_{\perp}$ rejection ratio is strongly dependent on the QWP rotation but nearly independent of the bias retardation, while the lower right panel shows that $\mathrm{LD}_{\times}$is almost independent of the QWP rotation but strongly dependent on the bias retardation. This result indicates that bias retardation and QWP rotation can be used to tune the rejection ratio of $\mathrm{LD}_{\perp}$ and $\mathrm{LD}_{\times}$independently.

Experimental Results. The experimental calibration scheme of the rejection ratio is illustrated in Figure 1. By inserting the calibration insert we can quantify and tune the setup's experimental rejection ratio of LD as described in the Supporting Information (Figure S1). Using the calibration insert, we find that our setup has a LD rejection ratio of at least 200 at all angles of the polarizer, which for most samples we work with is a good enough value to exclude any artifacts induced by LD. This means that if the object we want to study exhibits an LD that is 200 times stronger than its CD, then the artifact signal generated by the cross talk would be as large as the intrinsic CD signal. Our calibration experiments yielded a rejection ratio roughly 1 order of magnitude worse than the rejection ratios we find in our simulations $\left(\sim 10^{3}\right)$. We attribute this discrepancy to the finite beam aperture of the heating laser beam, thereby resulting in spatially inhomogeneous phase retardation of the heating beam in the EOM. In later PT measurements with Koehler illumination, however, as we only use the center of the heating beam to excite the small nanoparticle, the real rejection ratio is expected to be significantly better than the rejection ratio found upon calibration.

Aluminum Nanorods. To demonstrate the strength of our technique, especially regarding the distinction between $\mathrm{CD}$ and $\mathrm{LD}$, we have performed measurements on a purposetailored sample. The sample consists of nanofabricated aluminum nanorods, designed such that they absorb the 532 nm pump laser strongly along their long axis but weakly along the short axis, ${ }^{24}$ thereby exhibiting a strong LD. Aluminum NRs have higher LD $g$-factors than gold ones at our heating wavelength of $532 \mathrm{~nm}$ because the UV interband transitions of 

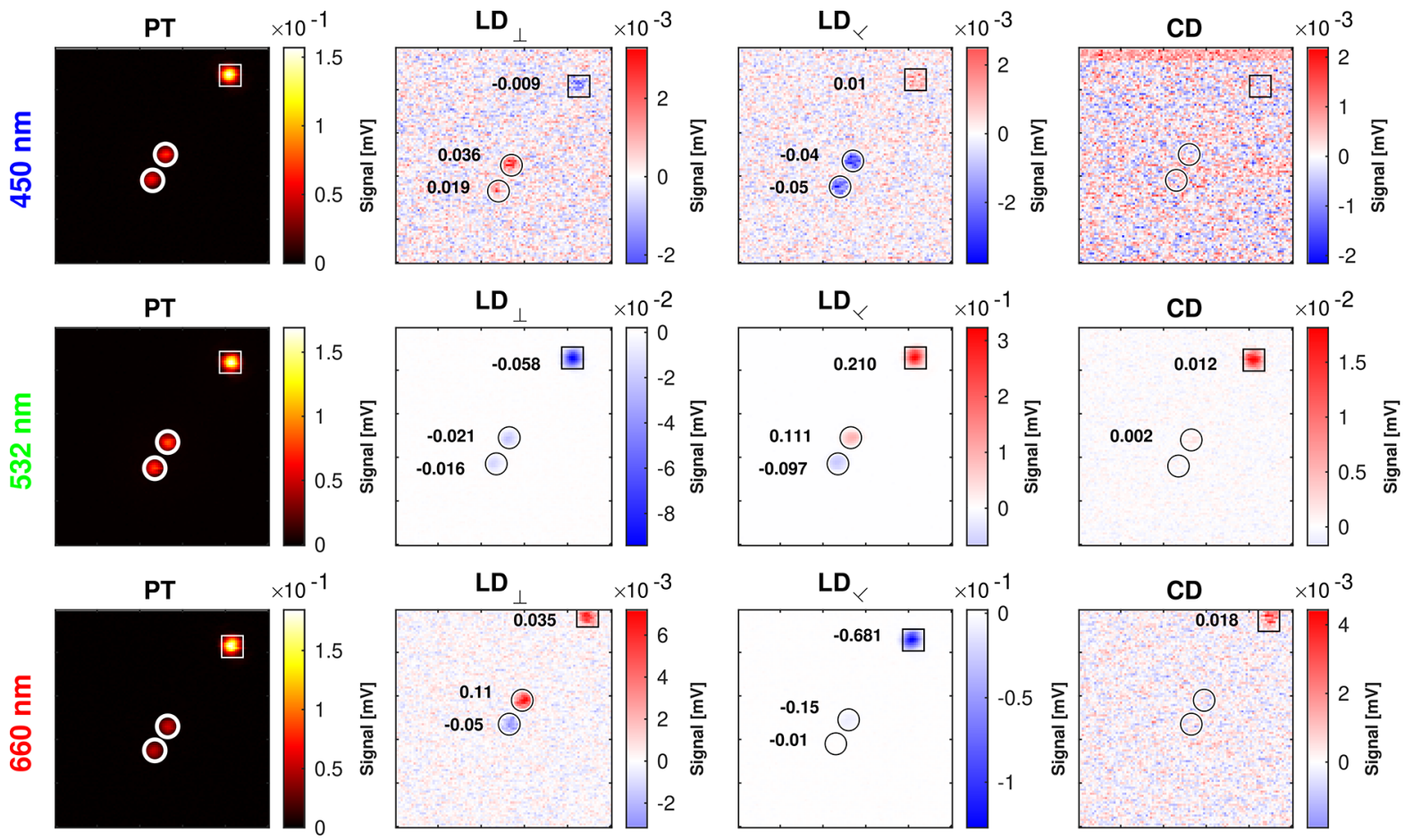

Figure 4. Photothermal (isotropic absorption), $\mathrm{LD}_{\perp}, \mathrm{LD}_{\times}$, and $\mathrm{CD}$ measurements of spherical gold nanoparticles at three different wavelengths $(450,532$, and $660 \mathrm{~nm})$ of the heating beam. The numbers indicated in $\mathrm{LD}_{\perp}, \mathrm{LD}_{\times}$, and $\mathrm{CD}$ images are the averaged $g$-factors for each spot. A histogram of CD values for several particles is displayed in Figure S6. The brightest particle in the PT image (square box in the upper right corner) is a dimer or trimer of nanoparticles (see Figure S5 for more CD and LD measurements on such a dimer). The probe beam intensity was $6 \mathrm{~mW}$ throughout the entire experiment. The respective heating powers were $0.6 \mathrm{~mW}$ at $450 \mathrm{~nm}, 5 \mathrm{~mW}$ at $532 \mathrm{~nm}$, and 8 $\mathrm{mW}$ at $660 \mathrm{~nm}$, measured at the entrance of the objective.

aluminum do not damp the visible plasmon resonance, in contrast to the case of gold. ${ }^{25}$ The rods are immersed in toluene to provide high PT contrast. The measurement results are shown in Figure 3. We find that the NRs exhibit the expected large $\mathrm{LD}_{\perp}$ and $\mathrm{LD}_{\times}$amplitudes and that the $\mathrm{CD}$ signal amplitude, if there is any, is buried below the noise. This result proves that our method can discriminate the effect of $\mathrm{CD}$ and LD with virtually no cross talk, thereby facilitating the artifact-free measurement of samples with very weak CD. We find a rejection ratio of $313 \pm 9$ for $\mathrm{LD}_{\perp}$ and $340 \pm 11$ for $\mathrm{LD}_{\times}$, respectively. As mentioned above, these values are significantly higher than the one (200) found upon calibration and are consistent with our calibration measurement using the entire beam aperture, whereas the nanoparticle measurement only makes use of the very center of the beam, presumably with smaller polarization imperfections. Here we assume that the NRs are perfectly achiral objects. Although this assumption holds true for ideal nanorods, fabrication errors could give rise to residual CD. In our CD measurements we do not find any measurable CD signal. On the basis of the SNR, we can therefore give an upper bound of $3 \times 10^{-3}$ for the $g$-factor of these rods.

$C D$ and $L D$ Measurements at Three Different Wavelengths. So far, we have shown $\mathrm{CD}$ measurements only at a single wavelength. To retrieve useful structural information on a sample of interest one should record its full CD spectrum. As we had no access to a tunable heating laser with the required power, we instead used three lasers at different wavelengths, 450,532 , and $660 \mathrm{~nm}$, to show that our technique is compatible with spectral measurements. Although the EOM's retardance is strongly dependent on the beam parameters (width and pointing angles), we found our alignment method robust enough to overlap the different laser beams by means of flippable mirrors, while still keeping excellent rejection ratios. Figure 4 shows measurements of $100 \mathrm{~nm}$ diameter quasispherical gold nanoparticles at three different wavelengths. For each wavelength we performed a full analysis of the absorption of the nanoparticles including isotropic absorption, linear dichroism, and circular dichroism. The scans shown in Figure 4 contain measurements of two single particles and one small cluster of particles. At a heating beam wavelength of $450 \mathrm{~nm}$, we find the particles to have both weak linear dichroism and weak circular dichroism. This observation fits with our expectation that the interband transitions of gold strongly damp plasmonic behavior at that wavelength and that polarization dependence is weak at this wavelength. We find relatively strong linear dichroism for both 532 and $660 \mathrm{~nm}$ heating wavelengths. This residual $\mathrm{LD}$ can be explained by small protrusions of the particle along one or more axes that may have formed during synthesis. Depending on the axis along which the particle is excited, one observes a different plasmon resonance wavelength and therefore linear dichroism. Notably, the particle with the strongest PT signal most likely corresponds to a dimer or trimer of nanoparticles. This cluster has a much stronger $g$-factor in LD than the single particles. If two nanoparticles are very close together, then they couple plasmonically. The degeneracy of the plasmons of the two nanoparticles may be lifted to yield two longitudinal modes, a symmetric and an antisymmetric mode, whereas the transverse plasmon resonances are not much affected. The symmetric longitudinal mode can be considerably red-shifted from the transverse mode. ${ }^{26}$ This plasmon coupling can lead to the 

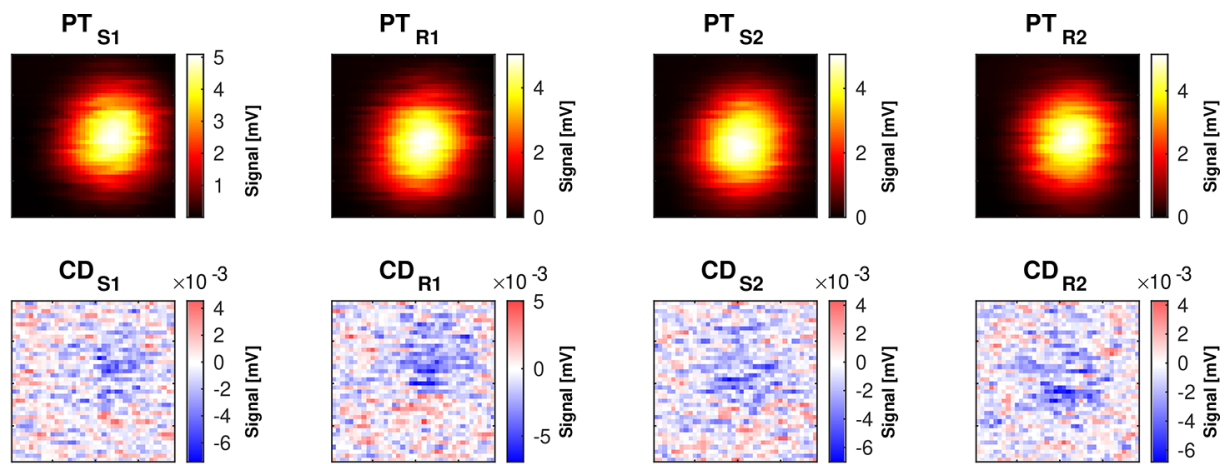

S1

R1

S2

R2

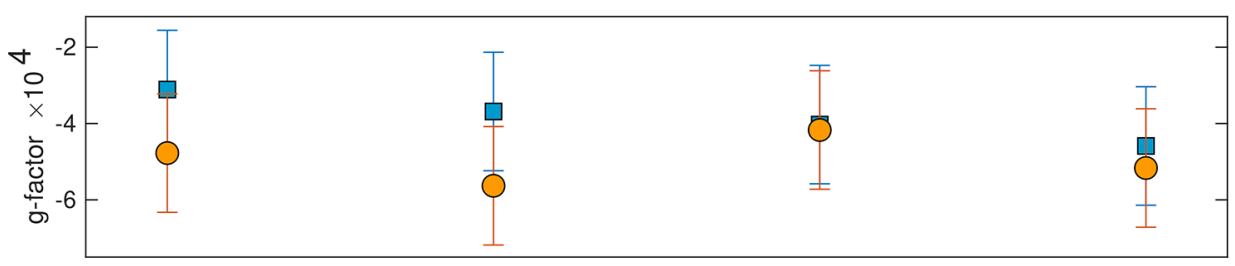

Figure 5. Photothermal (top) and photothermal circular dichroism (middle) measurement of a single $100 \mathrm{~nm}$ diameter spherical gold nanoparticle immersed in the two pure enantiomers of the carvone molecule ( $S$-carvone and $R$-carvone). (bottom) Corresponding $g$-factors of the scans. We employed two methods to calculate the $g$-factor. For the blue data points we use all pixels of the scan, for the orange data points we use a $20 \times 20$ pixel $^{2}$ array around the centroid position of the corresponding PT scan. The heating beam intensity was $17 \mathrm{~mW}$ and the probe beam intensity was $2.5 \mathrm{~mW}$.

relatively strong LD in our measurements at both wavelengths. The images of the single particles are barely visible in the CD scans, but the cluster shows a clear CD signal, which probably also arises from plasmonic coupling. ${ }^{27,28}$ The limited power of the $450 \mathrm{~nm}$ and the $660 \mathrm{~nm}$ lasers in these measurements prevented us from measuring $g$-factors smaller than $10^{-3}$. From the strength of the PT signal at $450 \mathrm{~nm}$ and from the low CD value, we hypothesize that the cluster is composed of two or three particles. These results prove that our technique can in principle be extended to obtain the spectral dependence of $\mathrm{CD}$, provided one has access to a tunable laser with sufficient power $(\sim 10 \mathrm{~mW}$ in the sample plane). The main factor that could hamper compatibility with spectral measurements in our technique is the wavelength dependence of the retarding elements. By using Koehler illumination for the heating beam, the technique is relatively insensitive to chromatic aberrations of the lenses. However, to ensure good cancellation of cross talk between $\mathrm{LD}$ and $\mathrm{CD}$, it is of the utmost importance to ensure the correct retardance of the polarization optics. Both the EOM's and the PEM's retardances can be adjusted easily to deliver the required maximum modulation phase of $\pi / 2$ for each wavelength. The QWP in use, although it is a superachromatic one, exhibits a slight dependence of its retardance on wavelength. One solution would be to compensate the wavelength dependence by making use of a liquid crystal retarder with adjustable retardance. We found that by combining the aforementioned slight rotation of the QWP with an adjustment of the EOM bias retardation it is possible to use a superachromatic or simply achromatic waveplate while achieving a similar rejection of LD-to-CD cross talk. Thanks to its good sensitivity and to its compatibility with spectral measurements, we foresee the great potential of our technique in the study of single plasmonic particles, including those having $\mathrm{CD}$ and $\mathrm{LD}$ bands in the near-IR range. A supercontinuum laser or a Ti:sapphire laser could conveniently provide a large spectral range while still delivering enough heating power for measuring CD $g$-factors down to the few $10^{-4}$ level. Heating in the near-IR would best be combined with a probe at an even more red-shifted wavelength. We think that the technique is compatible with a transmission geometry, ${ }^{29,30}$ which would allow one to probe in the mid-IR. In that case, one can use a near-IR-optimized refractive objective for heating and a reflective objective for the probing. Compared to more conventional techniques based on scattering or extinction, our absorption-based detection can measure CD signals of single nanoparticles with excellent signal-to-noise ratio., ${ }^{5,12,31}$

Gold Nanoparticles Immersed in a Chiral Liquid. We have characterized our setup's performance in terms of rejection of unwanted effects such as the leakage of linear dichroism or residual intensity modulation of the polarization modulators. These steps were necessary to make sure that we have the sensitivity to measure CD $g$-factors of single nanoparticles in the few $10^{-4}$ range, while at the same time avoiding cross talk induced by LD. In the experiments reported below, we wanted to investigate the very small circular dichroism of nominally achiral nanoparticles induced by a chiral liquid surrounding the particle. ${ }^{32}$ The effect, referred to as PCCD (plasmon-coupled circular dichroism), may facilitate the detection of chirality of biomolecules in the visible, where most biomolecules have no absorption band. ${ }^{8}$ According to the theoretical model presented in refs 32 and 33. PCCD has two components: one due to enhanced absorption of the molecule in the near field of the plasmonic particle and the second one due to chiral currents induced in the nanoparticle by the molecular dipole. In the present case, the chiral liquid does not absorb in the visible light range, and only the latter component is left, which corresponds to heat dissipation by the particle (at the plasmon resonance, the particle's absorption dominates molecular absorption anyway).

In their more recent work, Yoo et al. ${ }^{34}$ presented a solution of Maxwell's equations in circular bases, dubbed chiral Mie 
theory. They concluded that spherical gold nanoparticles, when immersed in a pure chiral liquid, can exhibit a measurable circular dichroism in the region of the particles' plasmon resonances. So far, none of the available experimental methods has been sensitive enough to detect the small PCCD signal of a single nanoparticle. Although Zhang et al. ${ }^{5}$ have demonstrated CD measurements on single clusters of nanorods, the sensitivity limit of their technique could not resolve CD $g$-factors far below $10^{-1}$. The sensitivity of photothermal $\mathrm{CD}$, however, reaches down to $g$-factors of a few $10^{-4}$, and might enable us to probe the magnitude of PCCD. To investigate the system proposed by Yoo et al., we spin-coated $100 \mathrm{~nm}$ spherical gold nanoparticles on a glass surface and glued this sample onto a half-open channel to form a flow cell. We then performed measurements on the same single nanoparticles with two different liquids as photothermal media. These liquids were the pure enantiomers of carvone (pure $S$-carvone and pure $R$-carvone.) For each liquid, we performed isotropic absorption measurements. Figure 5 top/ middle shows absorption and circular dichroism scans, respectively, of the same single nanoparticle surrounded by the pure enantiomers of opposite handedness, and Figure 5 bottom shows the corresponding calculated CD $g$-factors of each measurement. The measurements were carried out with a heating wavelength of $532 \mathrm{~nm}$.

We find that the particle initially exhibits weak CD (Figure 5 S1) when immersed in $S$-carvone. To find out whether this initial $\mathrm{CD}$ is due to geometric distortions of the particle itself or due to $\mathrm{CD}$ induced by the chiral liquid (PCCD) we flush the sample cell with $R$-carvone which is of opposite handedness and perform another set of absorption measurements. The PT signal, which represents the isotropic absorption of the particle, remains practically unchanged (Figure 5 top, $\mathrm{S} 1$ and $\mathrm{R} 1$ ). If the initial $\mathrm{CD}$ signal was due to the chiral liquid, then its sign should change upon reversal of the liquid's handedness. We find, however (Figure 5 bottom), that the change of $\mathrm{CD}$ is negligible within noise. That means that the measured $\mathrm{CD}$ is due to geometric chirality of the particle itself and, more importantly, that the chiral liquid does not alter the particles CD by more than a $g$-factor of $3 \times 10^{-4}$. Repeating the experiment by measuring in both liquids again (measurements S2 and R2) reproduces our findings that the changes of $\mathrm{CD}$ signals are within noise. We confirm the absence of residual leakage of $\mathrm{LD}$ into $\mathrm{CD}$ by performing $\mathrm{LD}$ reference measurements which are shown in Figure S7. The residual leak of $L D$, considering our rejection ratio of 300 , is one magnitude smaller than the measured $C D$ signal and can therefore be safely neglected. We want to note here that the sensitivity of the measurements was limited due to heatinginduced reshaping of the particles. ${ }^{35-37}$ In order to avoid reshaping, we had to use heating powers below $20 \mathrm{~mW}$. Higher powers cause long-term changes of the PT signal as shown in Figure S8.

The results of Yoo et al. ${ }^{34}$ predict a differential absorption of about $10^{-2}$ for a liquid with a large circular birefringence (CB) of $10^{-2}$. The circular birefringence of carvone corresponds to a difference in refractive indices for circular polarization of about $10^{-4}$. $^{38,39}$ Scaling the results of Yoo et al. linearly with CB, we therefore expect an induced $\mathrm{CD}$ of about $10^{-4}$ in carvone, in good agreement with the estimations of ref 33 . This value is below, or close to our detection limit. Therefore, despite our improved sensitivity, we are not able to report any measurable PCCD effect for a gold nanoparticle in carvone heated with
$532 \mathrm{~nm}$. Reshaping of the particle, laser-induced chemical reactions, ${ }^{40}$ and instabilities or drifts of our optical setup set a limit to our detection sensitivity of PCCD (see the Supporting Information section "Heat-induced reshaping").

To improve on this result, one could consider using other shapes of plasmonic particles, such as nanorods or nanocubes, which present a stronger dependence of absorption on the surrounding refractive index due to their sharper plasmon resonances. These particles, however, are even more prone to reshaping than quasi-spherical particles. ${ }^{35}$ The lower powers needed to avoid reshaping would lower the photothermal sensitivity. Another strategy would be to enhance PCCD by choosing a surrounding medium with a larger circular birefringence. Alternatively, we might be able to improve the measurement sensitivity further by employing more efficient contrast mechanisms, as recently demonstrated in refs 41-43. This would allow us to observe $g$-factors down to the $10^{-4}$ range while at the same time substantially decreasing the heating and therefore avoiding reshaping.

\section{CONCLUSIONS}

We have improved our previously reported PTCD microscope ${ }^{15}$ by the addition of dual modulation of the polarization and of a QWP. We achieved CD measurements with excellent rejection of artifacts induced by vibrations, residual amplitude modulation, and residual linear birefringence, and we removed leakage of linear dichroism into circular dichroism down to below our detection limit. Compared to our previous single square-wave modulation scheme, ${ }^{15}$ we suppressed these artifacts by nearly 2 orders of magnitude. Additionally, by means of a set of static birefringent elements, we can retrieve the full polarization dependence of a single particle's absorption. Our simulations provide a simple tool to understand how to optimize modulation parameters to avoid, or to compensate for, residual depolarizing effects of the optics. We have experimentally demonstrated the rejection of $\mathrm{LD}$ leakage into $\mathrm{CD}$ by more than a factor of 200 allowing us to measure weak $C D$ signals of single quasi-spherical gold nanoparticles with a high sensitivity down to $3 \times 10^{-4}$ in $g$ factors. Furthermore, we also show the capability of the method to perform spectral CD measurements. Studying nominally achiral plasmonic nanoparticles in a chiral liquid environment, we could not detect any significant plasmoninduced transfer of chirality from the liquid to the particle. We therefore find a PCCD upper bound of a $3 \times 10^{-4}$ for our sample at $532 \mathrm{~nm}$. This $g$-factor sensitivity applies to $100 \mathrm{~nm}$ gold particles. We can translate it into a difference of absorption cross section of $\sim 6 \mathrm{~nm}^{2}$, independent of particle size at a fixed heating power. Smaller gold particles, however, would allow higher heating intensities, giving rise to higher sensitivities. Plasmonic systems like single particles with more complex shapes or dimers of nanoparticles may turn out to be better candidates to investigate the weak PCCD effects.

\section{METHODS AND EXPERIMENTAL}

Sample Preparation. Single aluminum nanorods (AlNRs) were nanofabricated on a glass substrate using e-beam lithography. The glass substrate was sandwiched with a second cavity glass slide to contain a liquid for photothermal measurements. Toluene was used as the photothermal medium because of its high thermo-refractive coefficient. The $100 \mathrm{~nm}$ gold nanosphere samples were prepared by spin-coating a pure stock solution (nanoComposix) on a glass slide at 
$500 \mathrm{rpm}$ for $10 \mathrm{~s}$ followed by $4000 \mathrm{rpm}$ for $60 \mathrm{~s}$. This was followed by UV cleaning for $30 \mathrm{~min}$.

Experimental Setup. A schematic of the experimental setup is shown in Figure 1. The details about the setup can be found in our previous report. ${ }^{15}$ In comparison to our previous setup (which contained a single modulator, EOM), a second polarization modulator (PEM) was added to perform dual modulation of the polarization. The EOM and PEM were sinusoidally driven at modulation frequencies 23.5 and $50 \mathrm{kHz}$, respectively. In addition, we have used two circular birefringent plates, $\mathrm{CB}_{90}$ and $\mathrm{CB}_{45}$, which rotate the linear polarization by $90^{\circ}$ and $45^{\circ}$, respectively. The heating beam diameter was $\sim 1 \mathrm{~mm}$, when propagating through the polarization modulators

Optical and Electronic Equipment. EOM: Conoptics 350-80LA-01 (extinction ratio 300:1); EOM driver: Conoptics Model 302RM, PEM: Hinds Instruments PEM-100 ; objective: Olympus PLAPON60XOTIRFM-SP; wave-plate: Thorlabs AQWP05M-600; optical rotators: Edmund Optics no. 34-308 and no. 34-307; lockin: Stanford Research Systems Model SR844 RF; probe laser: SpectraPhysics Model 3900S; heating laser: Verdi V10 (also used as pump for the Spectra-Physics laser).

Data Analysis. The $g$-factors for each single gold nanopshere in Figure 4 were calculated as ratios of mean values of photothermal and photothermal circular and linear dichroism signals averaged in an area of $7 \times 7$ pixels $^{2}$ around the centroid of each spot. The $g$-factors for the gold nanosphere in Figure 5 were caclulated in areas of $20 \times 20$ and $38 \times 38$ pixels $^{2}$ around the centroid position, respectively. The rejection ratio of the aluminum nanorod measurements was calculated taking the mean value of a $5 \times 5$ pixels $^{2}$ area centered around the maximum values of the respective $\mathrm{LD}_{\perp}$ and $\mathrm{LD}_{\times}$measurements and dividing it by the noise standard deviation of the same region in the CD image.

\section{ASSOCIATED CONTENT}

\section{SI Supporting Information}

The Supporting Information is available free of charge at https://pubs.acs.org/doi/10.1021/acsnano.1c05374.

Stokes representation of polarization, experimental alignment procedure, alignment protocol, quantification of the extinction ratio, simulations, Jones matrix representation of the optical components, simulated FFT spectra of different absorbers, CD of gold nanoparticle dimers, histogram of CD $g$-factors of single nanoparticles, $C D$ of a single gold nanoparticle in carvone with LD reference, heat-induced reshaping, nanofabrication of single aluminum nanorods (PDF)

\section{AUTHOR INFORMATION}

\section{Corresponding Author}

Michel Orrit - Huygens-Kamerlingh Onnes Laboratory, Leiden University, Leiden 2300 Rapenburg, Netherlands; ๑ orcid.org/0000-0002-3607-3426; Email: orrit@ physics.leidenuniv.nl

\footnotetext{
Authors

Patrick Spaeth - Huygens-Kamerlingh Onnes Laboratory, Leiden University, Leiden 2300 Rapenburg, Netherlands; (1) orcid.org/0000-0001-8520-6216

Subhasis Adhikari - Huygens-Kamerlingh Onnes Laboratory, Leiden University, Leiden 2300 Rapenburg, Netherlands; (1) orcid.org/0000-0002-0914-433X

Martin Dieter Baaske - Huygens-Kamerlingh Onnes Laboratory, Leiden University, Leiden 2300 Rapenburg, Netherlands; ㅇo이.org/0000-0003-2384-7557
}

Sergii Pud - Huygens-Kamerlingh Onnes Laboratory, Leiden University, Leiden 2300 Rapenburg, Netherlands; (1) orcid.org/0000-0002-1393-9135

Jacco Ton - Huygens-Kamerlingh Onnes Laboratory, Leiden University, Leiden 2300 Rapenburg, Netherlands

Complete contact information is available at:

https://pubs.acs.org/10.1021/acsnano.1c05374

\section{Author Contributions}

${ }^{\ddagger}$ P.S. and S.A. contributed equally to this work.

\section{Notes}

The authors declare no competing financial interest.

\section{ACKNOWLEDGMENTS}

This work was supported by The Netherlands Organisation for Scientific Research (NWO/OCW), as part of the Frontiers of Nanoscience (NanoFront) program, and Open Technology Program (OTP, Project No. 16008). We thank Dr Wolfgang Löffler for providing optical equipment and Mr Laurent Le for helpful discussions. We thank Dr Wiebke Albrecht for carrying out TEM measurements.

\section{REFERENCES}

(1) Lombardi, R. A.; Nafie, L. A. Observation and Calculation of Vibrational Circular Birefringence: A New Form of Vibrational Optical Activity. Chirality 2009, 21, E277-E286.

(2) Hendry, E.; Mikhaylovskiy, R.; Barron, L.; Kadodwala, M.; Davis, T. Chiral Electromagnetic Fields Generated by Arrays of Nanoslits. Nano Lett. 2012, 12, 3640-3644.

(3) Tang, Y.; Cohen, A. E. Enhanced Enantioselectivity in Excitation of Chiral Molecules by Superchiral Light. Science 2011, 332, 333-336.

(4) Schäferling, M.; Dregely, D.; Hentschel, M.; Giessen, H. Tailoring Enhanced Optical Chirality: Design Principles for Chiral Plasmonic Nanostructures. Phys. Rev. X 2012, 2, 031010.

(5) Zhang, Q.; Hernandez, T.; Smith, K. W.; Hosseini Jebeli, S. A.; Dai, A. X.; Warning, L.; Baiyasi, R.; McCarthy, L. A.; Guo, H.; Chen, D.-H.; Dionne, J. A.; Landes, C. F.; Link, S. Unraveling the Origin of Chirality from Plasmonic Nanoparticle-Protein Complexes. Science 2019, 365, 1475-1478.

(6) Raziman, T.; Godiksen, R. H.; Müller, M. A.; Curto, A. G. Conditions for Enhancing Chiral Nanophotonics near Achiral Nanoparticles. ACS Photonics 2019, 6, 2583-2589.

(7) Solomon, M. L.; Abendroth, J. M.; Poulikakos, L. V.; Hu, J.; Dionne, J. A. Fluorescence-Detected Circular Dichroism of a Chiral Molecular Monolayer with Dielectric Metasurfaces. J. Am. Chem. Soc. 2020, 142, 18304-18309.

(8) Maoz, B. M.; Chaikin, Y.; Tesler, A. B.; Bar Elli, O.; Fan, Z.; Govorov, A. O.; Markovich, G. Amplification of Chiroptical Activity of Chiral Biomolecules by Surface Plasmons. Nano Lett. 2013, 13, $1203-1209$.

(9) García-Guirado, J.; Svedendahl, M.; Puigdollers, J.; Quidant, R. Enantiomer-Selective Molecular Sensing Using Racemic Nanoplasmonic Arrays. Nano Lett. 2018, 18, 6279-6285.

(10) Jellison, G.; Modine, F. Two-Modulator Generalized Ellipsometry: Theory. Appl. Opt. 1997, 36, 8190-8198.

(11) Jellison, G.; Modine, F. Two-Modulator Generalized Ellipsometry: Experiment and Calibration. Appl. Opt. 1997, 36, 8184-8189.

(12) Vinegrad, E.; Vestler, D.; Ben-Moshe, A.; Barnea, A. R.; Markovich, G.; Cheshnovsky, O. Circular Dichroism of Single Particles. ACS Photonics 2018, 5, 2151-2159.

(13) Novotny, L.; Hecht, B. Principles of Nano-Optics; Cambridge University Press: Cambridge, 2012.

(14) Bauer, T. Probe-Based Nano-Interferometric Reconstruction of Tightly Focused Vectorial Light Fields. Thesis, Friedrich-AlexanderUniversität Erlangen-Nürnberg, 2017. 
(15) Spaeth, P.; Adhikari, S.; Le, L.; Jollans, T.; Pud, S.; Albrecht, W.; Bauer, T.; Caldarola, M.; Kuipers, L.; Orrit, M. Circular Dichroism Measurement of Single Metal Nanoparticles Using Photothermal Imaging. Nano Lett. 2019, 19, 8934-8940.

(16) Whittaker, E. A.; Gehrtz, M.; Bjorklund, G. C. Residual Amplitude Modulation in Laser Electro-Optic Phase Modulation. J. Opt. Soc. Am. B 1985, 2, 1320-1326.

(17) Sathian, J.; Jaatinen, E. Intensity Dependent Residual Amplitude Modulation in Electro-Optic Phase Modulators. Appl. Opt. 2012, 51, 3684-3691.

(18) Shindo, Y.; Nakagawa, M. Circular Dichroism Measurements. I. Calibration of a Circular Dichroism Spectrometer. Rev. Sci. Instrum. 1985, 56, 32-39.

(19) Shindo, Y.; Nakagawa, M.; Ohmi, Y. On the Problems of CD Spectropolarimeters. II: Artifacts in CD Spectrometers. Appl. Spectrosc. 1985, 39, 860-868.

(20) Shindo, Y. On the Problems of CD Spectropolarimeter (IV) Artifacts Due to the Light Scattering by Small Particles. Appl. Spectrosc. 1985, 39, 713-715.

(21) Cheng, J.; Nafie, L.; Stephens, P. Polarization Scrambling Using a Photoelastic Modulator: Application to Circular Dichroism Measurement. J. Opt. Soc. Am. 1975, 65, 1031-1035.

(22) Barriel, O. A. Mueller Matrix Polarimetry of Anisotropic Chiral Media. Ph.D. thesis, Universitat de Barcelona, Barcelona, Spain, 2010.

(23) Arteaga, O.; Freudenthal, J.; Wang, B.; Kahr, B. Mueller Matrix Polarimetry with Four Photoelastic Modulators: Theory and Calibration. Appl. Opt. 2012, 51, 6805-6817.

(24) Knight, M. W.; Liu, L.; Wang, Y.; Brown, L.; Mukherjee, S.; King, N. S.; Everitt, H. O.; Nordlander, P.; Halas, N. J. Aluminum Plasmonic Nanoantennas. Nano Lett. 2012, 12, 6000-6004.

(25) Cheng, F.; Su, P.-H.; Choi, J.; Gwo, S.; Li, X.; Shih, C.-K. Epitaxial Growth of Atomically Smooth Aluminum on Silicon and Its Intrinsic Optical Properties. ACS Nano 2016, 10, 9852-9860.

(26) Jain, P. K.; El-Sayed, M. A. Plasmonic Coupling in Noble Metal Nanostructures. Chem. Phys. Lett. 2010, 487, 153-164.

(27) Shen, X.; Song, C.; Wang, J.; Shi, D.; Wang, Z.; Liu, N.; Ding, B. Rolling up Gold Nanoparticle-Dressed DNA Origami into ThreeDimensional Plasmonic Chiral Nanostructures. J. Am. Chem. Soc. 2012, 134, 146-149.

(28) Kuzyk, A.; Schreiber, R.; Fan, Z.; Pardatscher, G.; Roller, E.-M.; Högele, A.; Simmel, F. C.; Govorov, A. O.; Liedl, T. DNA-Based SelfAssembly of Chiral Plasmonic Nanostructures with Tailored Optical Response. Nature 2012, 483, 311-314.

(29) Berciaud, S.; Lasne, D.; Blab, G. A.; Cognet, L.; Lounis, B. Photothermal Heterodyne Imaging of Individual Metallic Nanoparticles: Theory versus Experiment. Phys. Rev. B: Condens. Matter Mater. Phys. 2006, 73, 045424.

(30) Selmke, M.; Braun, M.; Cichos, F. Photothermal Single-Particle Microscopy: Detection of a Nanolens. ACS Nano 2012, 6, 27412749.

(31) Narushima, T.; Okamoto, H. Circular Dichroism Microscopy Free from Commingling Linear Dichroism via Discretely Modulated Circular Polarization. Sci. Rep. 2016, 6, 35731.

(32) Govorov, A. O.; Fan, Z.; Hernandez, P.; Slocik, J. M.; Naik, R. R. Theory of Circular Dichroism of Nanomaterials Comprising Chiral Molecules and Nanocrystals: Plasmon Enhancement, Dipole Interactions, and Dielectric Effects. Nano Lett. 2010, 10, 1374-1382.

(33) Govorov, A. O.; Fan, Z. Theory of Chiral Plasmonic Nanostructures Comprising Metal Nanocrystals and Chiral Molecular Media. ChemPhysChem 2012, 13, 2551-2560.

(34) Yoo, S.; Park, Q.-H. Enhancement of Chiroptical Signals by Circular Differential Mie Scattering of Nanoparticles. Sci. Rep. 2015, 5, 14463.

(35) Albrecht, W.; Deng, T.-S.; Goris, B.; van Huis, M. A.; Bals, S.; van Blaaderen, A. Single Particle Deformation and Analysis of SilicaCoated Gold Nanorods before and after Femtosecond Laser Pulse Excitation. Nano Lett. 2016, 16, 1818-1825.
(36) Ruijgrok, P.; Verhart, N.; Zijlstra, P.; Tchebotareva, A.; Orrit, M. Brownian Fluctuations and Heating of an Optically Aligned Gold Nanorod. Phys. Rev. Lett. 2011, 107, 037401.

(37) Taylor, A. B.; Siddiquee, A. M.; Chon, J. W. Below Melting Point Photothermal Reshaping of Single Gold Nanorods Driven by Surface Diffusion. ACS Nano 2014, 8, 12071-12079.

(38) Lambert, J.; Compton, R.; Crawford, T. D. The Optical Activity of Carvone: A Theoretical and Experimental Investigation. J. Chem. Phys. 2012, 136, 114512.

(39) Reinscheid, F.; Reinscheid, U. Stereochemical Analysis of (+)-Limonene Using Theoretical and Experimental NMR and Chiroptical Data. J. Mol. Struct. 2016, 1106, 141-153.

(40) Gaiduk, A.; Ruijgrok, P. V.; Yorulmaz, M.; Orrit, M. Making Gold Nanoparticles Fluorescent for Simultaneous Absorption and Fluorescence Detection on the Single Particle Level. Phys. Chem. Chem. Phys. 2011, 13, 149-153.

(41) Li, M.; Razumtcev, A.; Yang, R.; Liu, Y.; Rong, J.; Geiger, A. C.; Blanchard, R.; Pfluegl, C.; Taylor, L. S.; Simpson, G. J. FluorescenceDetected Mid-Infrared Photothermal Microscopy. J. Am. Chem. Soc. 2021, 143, 10809-10815.

(42) Zhang, Y.; Zong, H.; Zong, C.; Tan, Y.; Zhang, M.; Zhan, Y.; Cheng, J.-X. Fluorescence-Detected Mid-Infrared Photothermal Microscopy. J. Am. Chem. Soc. 2021, 143, 11490-11499.

(43) Ding, T. X.; Hou, L.; Meer, H. v. d.; Alivisatos, A. P.; Orrit, M. Hundreds-Fold Sensitivity Enhancement of Photothermal Microscopy in Near-Critical Xenon. J. Phys. Chem. Lett. 2016, 7, 2524-2529. 